\title{
Mode transformations and entanglement relativity in bipartite gaussian states
}

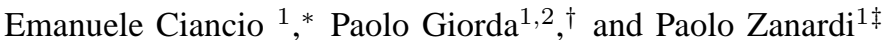 \\ ${ }^{1}$ Institute for Scientific Interchange (ISI), Viale Settimio Severo 65, 10133 Torino, Italy and \\ 2 Dipartimento di Fisica del Politecnico, C.so Duca degli Abruzzi 24, I-10129 Torino, Italy
}

(Dated: April 18, 2018)

\begin{abstract}
A proper choice of subsystems for a system of identical particles e.g., bosons, is provided by second-quantized modes i.e.,creation/annihilation operators. Here we investigate how the entanglement properties of bipartite gaussian states of bosons change when modes are changed by means of unitary, number conserving, Bogolioubov transformations. This set of "virtual" bi-partitions is then finite-dimensionally parametrized and one can quantitatively address relevant questions such as the determination of the minimal and maximal available entanglement. In particular, we show that in the class of bipartite gaussian states there are states which remain separable for every possible modes redefinition, while do not exist states which remain entangled for every possible modes redefinition
\end{abstract}

\section{INTRODUCTION}

The physical notion of quantum entanglement lies at the very heart of the novel and rapidly developing discipline of quantum information science [1]. The mathematical definition of entanglement requires the existence of a tensor product structure over the Hilbert state-space of the system $S$ under investigation; physically a multi-partition of $S$ into subsystems has to be identified. It is nearly obvious that this latter choice is by far not unique; in most of the cases there is actually a enormous set of potential multi-partitions available. Unless a physical, possibly problem dependent selection criterion is somehow provided, all those "virtual" multi-partitions of $S$ into subsystems are conceptually on the same foot.

In Refs [2] and [3] an operationally motivated framework for relativity of entanglement, based on operator-algebras of observables has been introduced. There, the key physical idea is that the relevant multi-partition is selected on the basis of the set of implementable quantum dynamics, including both unitaries and measurements, in the actual operational scenario. Mathematically, the necessary tensor product structure is induced over the global set space by a bunch of implementable commuting algebras of observables satisfying suitable natural properties [3]. Each of this algebras becomes then a local one i.e., it plays the role of the observable algebras of one of the "virtual" subsystems.

A very important class of physical systems is provided by the ensembles of identical particles. For this kind of systems, in view of the (anti)symmetrization postulates used to enforce the proper statistical behaviour, a tensor product structure of the physical state-space e.g., the totally symmetric subspace for bosons, is not naturally given. Accordingly, the extension of the notion of quantum entanglement to systems of indistinguishable particle is not a totally trivial task and it indeed gave raise to different, complimentary approaches [4], [5]. In this paper we shall follow the second-quantized approach where the subsystems are associated to modes [4], rather than to par-

* ciancio@isiosf.isi.it

łzanardi@isiosf.isi.it ticles [5]. We will focus on bi-partite bosonic gaussian states and we will study how entanglement changes under unitary, number-conserving, redefinition of modes i.e., subsystems. Gaussian states play a central role in the theory of entanglement for continuous variables systems; the reason is two-fold. First their experimental relevance; indeed most of the states of light, which are easily obtainable in the lab by means of linear optics devices e.g., beam splitters, are gaussian. Second, they are mathematically easy to handle. In spite of the fact that gaussian states live in the infinite-dimensional bosonic Fock space all of their physical features are indeed captured by the so-called Covariance Matrix $V$. From this finite-dimensional matrix one is able to extract straightforwardly various measures of entanglement like negativity and logarithmic negativity [6]. To this aim it is often useful to cast the covariance matrix in standard form $V_{s}$. This is possible via local operations which do not change the entanglement of the state [7].

The main goal of our work is to focus on the idea of entanglement relativity [2, 3] as it can be applied in the field of gaussian states. As already pointed out, once a gaussian state is given in terms of its covariance matrix with respect to a given tensor product structure, we study how the entanglement properties of this state change when new tensor product structures are defined via number conserving modes transformations. The latter will be defined in detail in the following sections. It is important to emphasize that this paper is not primarily aimed at studying gaussian states per se. The latter are used, thanks to their structural simplicity, as an ideal arena to address and illustrate in a quantitative way the somewhat abstract idea of entanglement relativity. It is important to stress the difference, with respect to the existing literature, of the conceptual status of the modes transformations that we are going to consider. Indeed, in most of the former studies (e.g., [8]), modes transformations are physically enacted by optical devices (e.g., beam-splitters) that actually change the quantum state under examination. In this paper instead, the quantum state $\rho$ of two bosonic modes, has to be thought as unchanged: it is just the way $\rho$ is decomposed into subsystems i.e., modes, that is modified. The situation is somewhat reminiscent of the difference between "active" and "passive" rotations in elementary geometry; just as in that case, while the mathematical objects involved can be the same, the conceptual interpretation is quite different. 
The paper is organized as follows. In Sect. (III) the basic ideas of entanglement relativity are reviewed. In Sect. (III) theoretical background on gaussian states is provided. Sects (IVA) and (IVB) are devoted to the analysis of pure and mixed gaussian states respectively. Finally Sect. (V) contains some discussions and conclusions.

\section{ENTANGLEMENT RELATIVITY}

In the general setting [3] the local commuting algebras we are going to consider in the following are those generated by the mode creation/annihilation operators [4]; the global statespace considered here is the Fock space (total number of particle unconstrained) that it is known to be isomorphic to the tensor product of harmonic oscillator state spaces. It is an elementary fact that, by using canonical transformations, one can move from a given set of modes to a new one defining a new tensor product structure i.e., new subsystems. One is then naturally led to analyze how the entanglement properties of a given quantum state $\rho$ depend on the choice of the set of modes and how they change when a different set of modes is considered. In particular, once $\rho$ is given, one may want to know whether it exists a choice of modes i.e., a tensor product structure, with respect to which $\rho$ is a separable state. In the negative case one may refer to such a state as absolutely entangled. Another natural task is to find the minimum and the maximum of the entanglement of $\rho$ over the set of all possible tensor product structures (i.e, modes redefinitions). Besides its theoretical interest this might have some practical relevance. For instance, the latter analysis allows one to identify for any given state the modes that correspond to the maximal entanglement and that can be fruitfully exploited for some quantum information processing protocol e.g., teleportation.

More formally, if one denotes by $\mathcal{T} \in \mathcal{T P S}$ one of all the possible tensor product structures (TPS) and by $E_{\mathcal{T}}$ an entanglement measure e.g., negativity, associated to the TPS $\mathcal{T}$ one can define at least two natural "absolute" i.e., TPSindependent, entanglement measures

$$
\begin{aligned}
& E^{+}(\rho):=\sup _{\mathcal{T} \in \mathcal{T} \mathcal{P} \mathcal{S}} E_{\mathcal{T}}(\rho), \\
& E^{-}(\rho):=\inf _{\mathcal{T} \in \mathcal{T} \mathcal{P} \mathcal{S}} E_{\mathcal{T}}(\rho) .
\end{aligned}
$$

The set of absolutely separable [18] and absolutely entangled states would then correspond respectively to $\left\{\rho / E^{+}(\rho)=0\right\}$ and $\left\{\rho / E^{-}(\rho)>0\right\}$. As mentioned above a very natural question that arises concerns the relative weight of these two subsets in full state space. Of course, a more pratically meaningful definition of a TPS-independent entanglement measure would involve in Eq. (1) a restricted set of operationally meaningful TPSs. Indeed, it should be clear that the definitions (1) will in general involve optimization procedures over a vast set of possibilities.

In order to make this program amenable to a quantitative treatment, in this paper we shall consider bi-partite gaussian states only. This allows us to focus on a subset of $\mathcal{T P S}$ whose elements are parametrized by finite-dimensional objects i.e., $N$ by $N$ unitary matrices.

\section{THEORETICAL BACKGROUND}

\section{A. Gaussian states}

Gaussian states are defined as those quantum states whose Wigner function is gaussian; they are completely defined by their second statistical moments. By grouping all the components $x_{i}$ of the position and momentum operators in a single column vector such that $x_{i}=q_{i}, x_{i+1}=p_{i}$, one can write [9]:

$$
W(x)=\left(4 \pi^{2} \sqrt{\operatorname{det} V}\right)^{-1} \exp \left[-\frac{1}{2}\left(x^{t} V^{-1} x\right)\right]
$$

where $W(x)$ is the Wigner function and $V$ is the covariance matrix. The entries of the latter are exactly all the second order statistical moments of the components of the canonical operators (position and momentum).

In general, in terms of the components $x_{i}$, the classical covariance is defined as: $\left.\left.\left\langle x_{i} x_{j}\right\rangle-<x_{i}\right\rangle<x_{j}\right\rangle$. Dealing with quantum operators the products appearing in the covariance expression must be taken in the Wigner-Moyal form, i.e. they must be symmetrized: $\left.\left\langle x_{i} x_{j}\right\rangle=<x_{i} x_{j}+x_{j} x_{i}\right\rangle / 2$. Since we work with gaussian states, the first moments (mean values) can be set to zero by means of displacement operators that do not change the entanglement properties [10]. Therefore $V$ is built by simply setting: $V_{i j}=<x_{i} x_{j}>$. In this way, the covariance matrix $V$ has all the variances $\left\langle x_{i}^{2}>\right.$ on the diagonal and the covariances $\left\langle x_{i} x_{j}\right\rangle, i \neq j$ off diagonal. Due to the Wigner-Moyal symmetrization $V$ turns out to be real and symmetric. In the following we will deal with bipartite systems: the identification of $x_{1}, x_{2}$ with $q_{A}, p_{A}$ and of $x_{3}, x_{4}$ with $q_{B}, p_{B}$, allows us to write $V$ in a block form:

$$
V=\left(\begin{array}{cc}
A & C \\
C^{t} & B
\end{array}\right)
$$

where $A, B$ refers to subsystem $\mathrm{A}($ lice $)$ and $\mathrm{B}(\mathrm{ob})$ respectively.

By using the covariance matrix formalism the Heisenberg uncertainty principle can be recast in the following compact way:

$$
V \geq \frac{i}{2} \sigma,
$$

where $\sigma$ is the so-called symplectic unity for two subsystems:

$$
\sigma=\left(\begin{array}{cccc}
0 & 1 & 0 & 0 \\
-1 & 0 & 0 & 0 \\
0 & 0 & 0 & 1 \\
0 & 0 & -1 & 0
\end{array}\right)
$$

Here, the uncertainty principle is expressed in its extended form; together with the variance relations, also the covariance relations are taken into account: $\operatorname{Var}\left[x_{i}\right] \operatorname{Var}\left[x_{j}\right]-$ $\operatorname{Cov}\left[x_{i}, x_{j}\right]^{2} \geq 1 / 4$. 
Any covariance matrix $V$ can be put in the so called standard form $V_{s}$ by means of a suitable local symplectic transformation (see below). In $V_{s}$ the blocks have a diagonal form [7]:

$$
V_{s}=\left(\begin{array}{cccc}
a & 0 & c_{+} & 0 \\
0 & a & 0 & c_{-} \\
c_{+} & 0 & b & 0 \\
0 & c_{-} & 0 & b
\end{array}\right)
$$

Since the transformation that gives $V_{s}$ is local, it does not change the entanglement properties of the state.

\section{B. Modes transformations}

A generic bosonic bipartite (gaussian) state can be described in terms of two harmonic oscillator modes. In a quantum system the operators accounting for the oscillation amplitudes are the annihilation/creation operators $a_{i}, a_{i}^{\dagger}$. The two subsystems corresponding to the bipartition are the two oscillation 'directions'. We can imagine Alice and Bob sharing the system, Alice having one mode, Bob having the other one. According to what we have already said about the relativity of the entanglement, one state can be entangled if described by a particular pair of modes and can be separable if described by a different pair. If we now consider how the modes can be changed we see that, from a phase-space point of view, the modes transformations correspond to canonical transformations of the conjugated (position and momentum) hamiltonian variables. The new variables must of course fulfill the same canonical commutation relations. The set of these canonical transformations form the symplectic non-compact group: $\operatorname{Sp}(2 n, \mathcal{R})$.

A well known class of mode transformations are the so called Bogolioubov transformations: $a \rightarrow b, \quad b_{i}=$ $\sum_{j} \alpha_{i j} a_{j}+\beta_{i j} a_{j}^{\dagger}$, with $i, j=1, \ldots, n$. For bipartite states $n=2$. A special sub-class of Bogolioubov transformations is given by:

$$
b_{i}=\sum_{j} U_{i j} a_{j}, \quad(i, j=1,2)
$$

where $U$ is a unitary $2 \times 2$ complex matrix. This kind of mode transformations are those which conserve the number of particles and are precisely the ones we are going to focus on in our analysis. These mappings form a group $K(n)$ that coincides with the compact subgroup of $\operatorname{Sp}(2 n, \mathcal{R})$ of maximal dimension [11]. Each mapping (7) induces a specific tensor product structure $\mathcal{T} \in \mathcal{T} \mathcal{P} \mathcal{S}$. The goal of our paper is then to study how entanglement varies when the modes redefinitions belonging to $K(n)$ are applied to a given state. In particular this means that in the remainder of the paper the ensemble $\mathcal{T P S}$ used to evaluate 10 is in a one-to-one correspondence with elements of $K(n)$.

Each of the unitary and symplectic transformations $S_{U} \in$ $K(n)$, where $U$ is defined in (7), can be written as [21]:

$$
S_{U}=\left(\begin{array}{cc}
X & -Y \\
Y & X
\end{array}\right)
$$

where $X$ and $Y$ are respectively the real and the imaginary part of the unitary $2 \times 2$ operator $U=X+i Y$ acting on the modes [11]:

$$
\begin{gathered}
X=\frac{U+U^{*}}{2} \\
Y=\frac{U-U^{*}}{2 i} .
\end{gathered}
$$

The covariance matrix transforms according to 8 as

$$
V^{\prime}=S_{U} V S_{U}^{t}
$$

We end this subsection by discussing how one can represent a general element of $K(n)$. In general a $2 \times 2$ unitary matrix $U$ depends on four real parameters; thus a possible and useful parametrization of $U$ is the following:

$$
U=\left(\begin{array}{cc}
\sin \theta e^{i \phi} & \cos \theta e^{i \phi^{\prime}} \\
\cos \theta e^{i \phi^{\prime \prime}} & \sin \theta e^{i\left(-\phi+\phi^{\prime}+\phi^{\prime \prime}-\pi\right)}
\end{array}\right)
$$

The latter is the parametrization we use in our numerical calculations.

\section{Entanglement measure}

In continuous variables systems a proper measure of entanglement that can be efficiently computed is logarithmic negativity [6, 19, 20]. Following Ref. [6] logarithmic negativity can be obtained starting from the trace-norm of the partially transposed density matrix. This trace-norm can be computed from the so-called symplectic eigenvalues of the partial transpose of $V$ with respect to the subsystem $A$, i.e. the eigenvalues of $\sigma^{-1} V^{T_{A}}$. The characteristic equation for $\sigma^{-1} V^{T_{A}}$ is:

$$
\lambda^{4}-(\operatorname{det} A+\operatorname{det} B-2 \operatorname{det} C) \lambda^{2}+\operatorname{det} V=0
$$

Thus the logarithic negativity can be computed as:

$$
E_{\mathcal{N}}=\frac{1}{2} \sum_{i} \max \left[-\log _{2}\left(2\left|\lambda_{i}\right|\right), 0\right] .
$$

\section{RESULTS}

In order to give a quantitative description of how the entanglement of a gaussian state changes under the action of all unitary transformations $S_{U} \in K(n)$, we have performed different kinds of numerical computations that now we briefly describe.

I. Pure states We start our analysis by considering pure gaussian states whose covariance matrix is in the standard form $V_{s}$. The modes transformations are generated by using a suitable discretization of the four dimensional parameters space defining $U$, see (11). For each transformation (mode decomposition) we compute the logarithmic negativity of the corresponding covariance matrix 10 .

Obviously the covariance matrices in the standard form do not describe all the possible gaussian states. $V_{s}$ is useful because it depends only on four parameters and has the same 
entanglement of a large class of states. Given a generic gaussian state and its covariance matrix $V$ it is easy to bring the latter in standard form by applying a suitable local symplectic transformation [7] $L \in S p(2, \mathcal{R}) \oplus S p(2, \mathcal{R})$ such that $V_{s}=L V L^{t}$. One can devise the following procedure to generate a set of generic $V$ s starting from the $V_{s}$ : given a covariance matrix $V_{s}$ one can apply the full set of local symplectic transformations $T \in S p(2, \mathcal{R}) \oplus S p(2, \mathcal{R})$; for each $T$ one obtains the matrix $\tilde{V}=T V_{s} T^{t}$ that corresponds to a squeezed state with the same entanglement of $V_{s}$ and with a possibly different energy. Once a generic $\tilde{V}$ has been generated, we apply the modes redefinition scheme $S_{U} \tilde{V} S_{U}^{t}$ and we evaluate the corresponding logarithmic negativity. The procedure is then repeated for many different $V_{s}$ and it is schematically displayed in Fig. (1).

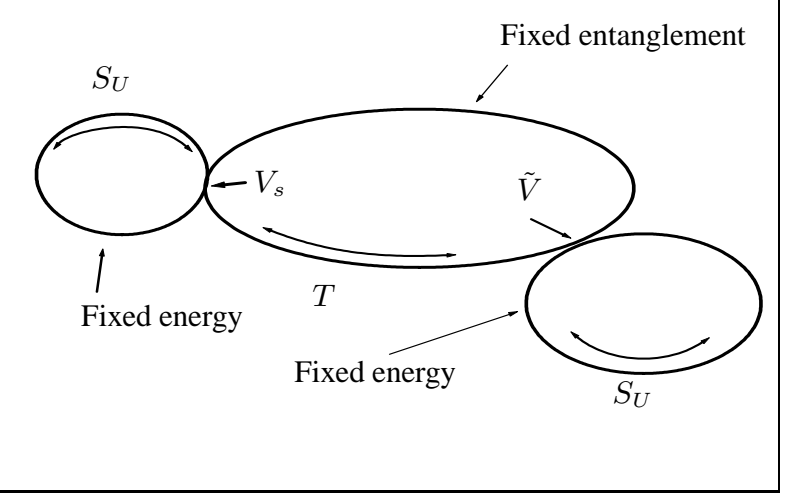

FIG. 1: A visual display of the procedure used to generate and decompose the gaussian states.

We would like to underline that while the $S_{U}$ s transformations (also referred in the literature as passive) are energy conserving, the $T$ s are entanglement conserving and that in general $S_{U} T \neq T S_{U}$. It is thus important to stress that applying the mode decomposition analysis to covariance matrices in non-standard form allows us to access a wider class of results. In this respect the present work differs from what has been done in several studies on gaussian state entanglement, e.g. [8, 12]. Those studies were indeed aimed at analyzing just entanglement properties of gaussian states, therefore the restriction to $V_{s}$ did not prevent the achievement of full generality. Here our aim is to study the variation of entanglement for different bipartitions associated with unitary modes redefinitions, no matter if the initial covariance matrix $V$ is $V_{s}$ or $\tilde{V}$. Thus the $T$ s transformations are merely used as a tool to define a more general set of new gaussian states; accordingly they do not have to be regarded as a new set of (number non-conserving) Bogolioubov transformations applied to the initial $V_{s}$."

II. Mixed states. A great number of mixed states have been generated in a random way by drawing the parameters $a, b$ and $c_{ \pm}$out of a uniform distribution over a well defined parameters range (see caption Fig. 7). Each (real symmetric) matrix is checked in order to verify whether it fulfills Heisenberg uncertainty principle; this allows one to select only the physical covariance matrices. The mode redefinition procedure is then applied and the maximal and minimal entanglement achievable is computed, see Eq. (1). These computations have involved covariance matrices both in standard and non-standard form.

In the following sections we describe in details the results of our computations.

\section{A. Pure states}

The first set of gaussian states we take into accountwhose covariance matrix is in the standard form $V_{s}$. In terms of $V_{s}$ they are described by only one independent parameter. In fact, with the usual notation (6), pure states correspond to covariance matrices with $a=b$ (pure states are always symmetric), $c_{ \pm}= \pm \sqrt{a^{2}-1}$, with the constraint $a \geq 1 / 2$. The pure gaussian states fulfill the Heisenberg uncertainty principle with the equal sign i.e., they minimize the uncertainty relations. We can consider two cases. In the first one $c=0$ : all covariance terms are zero and the corresponding states minimize the uncertainty relations in the usual form $\left(\Delta q_{i} \Delta p_{i}=1 / 2\right)$. In the second case, $c \neq 0$, the pure gaussian states corresponding to $V_{s}$ are the squeezed states [12]. It's not difficult to see that the non-squeezed states $(c=0)$ correspond to the isotropic oscillator case.

The results of the numerical computations show that :

$i$ ) there is always a unitary redefinition of the modes for which any pure state is separable i.e., $E^{-}(\rho)=0$;

ii) in particular, the non-squeezed states are absolutely separable: $E^{+}(\rho)=0$;

iii) in the case of squeezed states all unitary transformations lead to redefinitions of the modes (TPS) for which the value of the entanglement is always lower than value corresponding to the initial $V_{s}$.

A few comments are in order. The existence of a unitary redefinition of the modes for which the state is separable, see $i$ ), is not surprising. In fact this can be understood, by elementary arguments, in different ways. One one hand one can resort to the situation in which two coupled oscillators can be decoupled by the classical normal modes decomposition. A suitable choice of the mode operators (either classical or quantum) always allows one to deal with two independent mode of oscillation. On the other hand, the state is described in the configuration space by the Wigner function $W$ of Eq. (2). If we consider $W$ as a function of $q_{A}, q_{B}$ and a plane parallel to the $q_{A}, q_{B}$ plane intersecting $W\left(q_{A}, q_{B}\right)$, we get an ellipse whose axes lay on the bisectrices of the quadrants. In the isotropic case $\left(\left|c_{ \pm}\right|=0\right)$, see $\left.i i\right)$, the ellipse reduces to a circle, therefore the modes redefinitions, correspondig to rotations in the plane, do not change the variances of $q_{A}, q_{B}$. When $\left|c_{ \pm}\right|>0$ a covariance term appears and it is responsible for a non-vanishing excentricity of the ellipse, i.e. the gaussian Wigner function is 'squeezed'. A rotation (mode redefinition) of the reference axes always allows one to make them conincide with the ellipse's ones; therefore, it is always 
possible to set to zero the covariance term, which is responsible for the entanglement between the modes. In our case a $\pi / 4$ rotation reduces the entanglement to zero (see Fig. 2). In the $q_{A(B)} p_{A(B)}$ plane instead, since $\operatorname{Cov}\left[q_{A(B)}, p_{A(B)}\right]=0($ i.e., the blocks $A$ and $B$ in Eq. (3) are diagonal), the argument of Eq. (2) simply identifies a circle.

We now turn to examine point $i i i)$. In Fig. 2 the logarithmic negativity 13 of different pure sates $\rho$ is plotted as a function of $\theta\left(\phi, \phi^{\prime}, \phi^{\prime \prime}\right.$ in Eq. (11) are kept fixed).

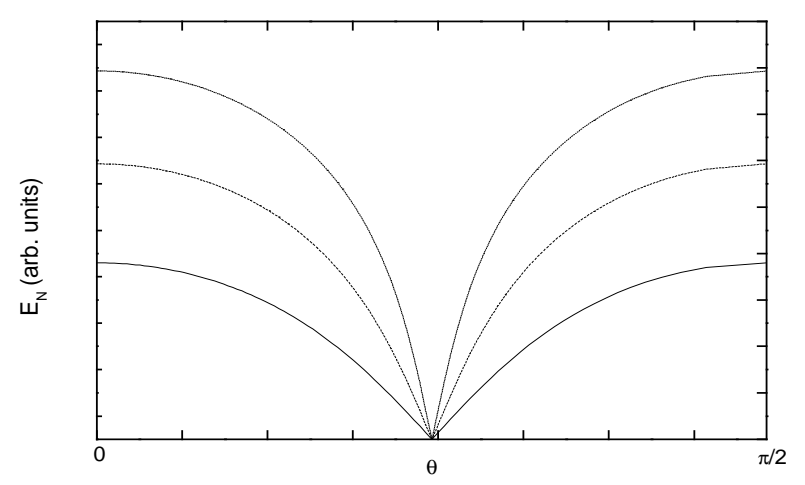

FIG. 2: Logarithmic negativity as function of the parameter $\theta$ for different pure states. The picture clearly shows a symmetric periodic behaviour. The point of zero entanglement is reached for $\theta=\pi / 4$ while the maxima are in correspondence of $\theta=0, \theta=\pi / 2$.

The maximal value of $E_{\mathcal{N}}$ i.e., $E^{+}(\rho)$, is achieved in correspondence of $\theta=0$ (modes swapping) and $\theta=\pi / 2$ (identity transformation). Any other value of $\theta$ produces a lower value of $E_{\mathcal{N}}$. In particular, for every state $\rho$, there is a value of $\theta$ for which $E_{\mathcal{N}}=0$. The results, that would not qualitatively change with a different choice of $\phi, \phi^{\prime}, \phi^{\prime \prime}$, can be understood as follows. In case of symmetric states the maximal entanglement is achieved in correspondence of $V_{s}$ because, in this case, all the quantum correlations between the operators are set to zero except those involving same-type operators between different subsystems. Every unitary modes redefinition gives rise to correlations between conjugated operators belonging to the same subsystem, while it decreases the correlation terms and consequently the entanglement between the two subsystems . Similar results were obtained in the context of optical passive transformations on gaussian states by Wolf et al.[8] and they are in agreement with the fact that the state described by $V_{s}$ corresponds, at given energy, to the maximum entanglement [13].

We now turn to discuss the states whose covariance matrix $\tilde{V}=T V_{s} T^{t}$ is not in standard form. It is important to realize that, at variance with the former studies of gaussian state entanglement, in the present setting the passage to the standard form is not harmless. In fact in order to cast a generic covariance matrix $V$ into its standard form $V_{s}$, one has to perform local operations $L$ ( e.g., local squeezing) that are outside the class of the modes redefinitions here allowed (8). Since these latter do not in general commute with the local operations $L$ required to get $V_{s}$ one obtains, as we will now see, rather different results depending whether the standard form is consid- ered or not.

The numerical computations, see Fig. 3 show that:

$i v)$ as described for the previous case, it is always possible to find a bipartition in which the state is separable i.e., $E^{-}(\rho)=0$;

$v$ ) it always exists a redefinition of the modes that can increase $E_{\mathcal{N}}$.

Fig. (4) shows the behaviour of the logarithmic negativity as a function of one of the local symplectic parameter (the squeezing operation) $\eta: a \mathbb{1} \rightarrow \operatorname{diag}\left(\eta a, \eta^{-1} a, \eta a, \eta^{-1} a\right)$ and of one of the global unitary transformation parameter $\theta$.

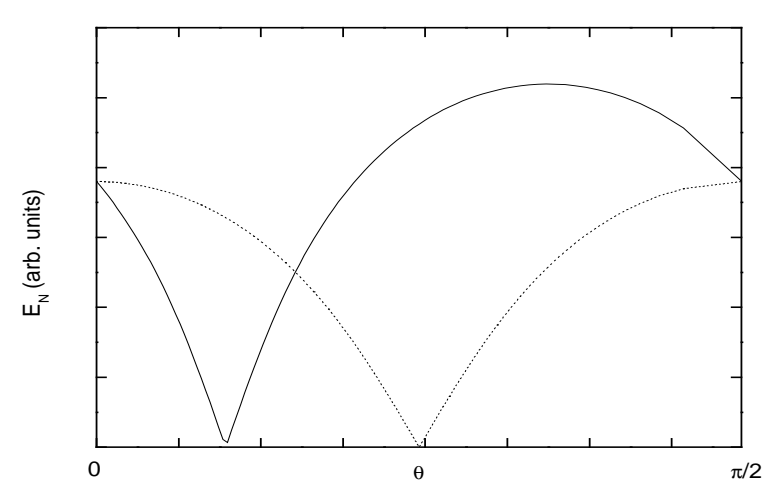

FIG. 3: The main difference between the behaviour of the pure state with $V_{s}$ (dotted line) and the one with $\tilde{V}$ (solid line) is the possibility for the latter to increase its logarithmic negativity

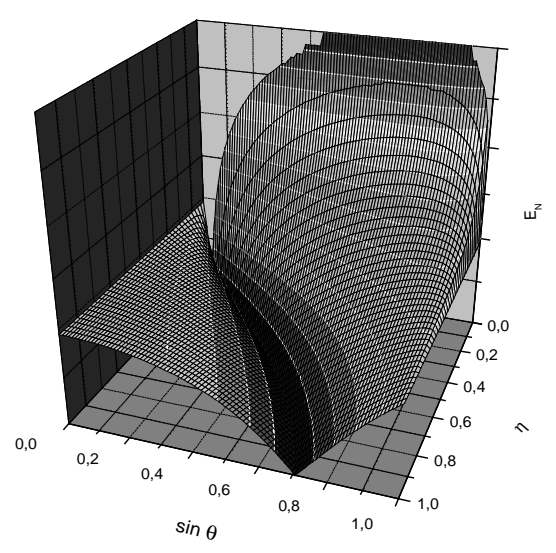

FIG. 4: A 3-dimensional plot of the logarithmic negativity as function of $\sin \theta$ and $\eta$. It is possible to note a line laying on the ground plane (dark valley) testifying the zero-entanglement modes redefinition for each initial state. Also it is worth noting the increasing value of $E_{\mathcal{N}}$ as $\eta$ gets different from 1, for some $U \neq \mathbb{1}$. 


\section{B. Mixed states}

The numerical analysis of mixed states leads to other interesting results. As in the previous section we start by considering the the covariance matrices in standard form. We first look at the symmetric $(a=b)$ subset of mixed states depending only on three parameters. If we set, as an additional condition, $c_{+}=-c_{-}$, we have states that behave in a way similar to the pure ones. In fact $E_{\mathcal{N}}$ is maximal in correspondence of the identity transformation and it decreases for all entangling $U \neq \mathbb{1}$. The difference consists in the fact that, instead of a single point, there is a whole interval of values of $\theta$ for which the logarithmic negativity vanishes, as displayed in Fig. 5] The further one moves away from pure states ( $p s)$ $\left(\left|c_{ \pm}\right|<\left|c_{ \pm}^{p s}\right|=\sqrt{a^{2}-1}\right)$ the wider is the plateau. When the plateau covers all the range of variation of $\theta$ the states become absolutely separable. The case $\left|c_{ \pm}\right|>\left|c_{ \pm}^{p s}\right|$ would be the only one for which there is no point of zero entanglement, but it has to be discarded since the corresponding matrices do not fulfill the Heisenberg principle. For simmetric mixed states such

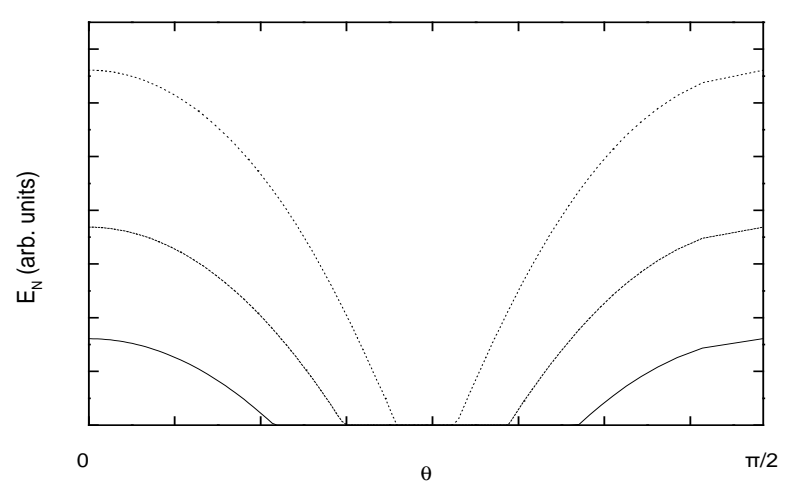

FIG. 5: The plot of the logarithmic negativity as a function of $\theta$ for three different symmetric mixed states shows a qualitatively similar behaviour for the three states. The width of the vanishing entanglement plateau is related to difference between $\left|c_{ \pm}\right|$and $\left|c_{ \pm}^{p s}\right|$

that $V \neq V_{s}$ the entanglement calculations give results that are similar to the ones obtained for the pure states case (see Fig 6): unitary redefinitions of the modes can increase the entanglement. In addition, as described above, $E_{\mathcal{N}}$ can vanish in correspondence of a whole interval of mode redefinitions.

Let us now turn to analyze the case of non-symmetric mixed states, starting as usual from those whose covariance matrices are in standard form. A great number of mixed states, in the defined range, turn out to be absolutely separable i.e., $E^{+}(\rho)=0$. Fig 7A shows the amount of absolutely separable states with respect to the amount of randomly generated physical states. Although the chosen range is not representative of the whole mixed gaussian states space i.e., different choices would have led to different results, it allows us to make some general remarks.

The high separability rate can be understood by using the Simon criterion [9]: it is sufficient for $c_{ \pm}$to have the same sign to achieve separability. This set of absolutely separable mixed states includes all the classical states defined in Ref. [14, 15].

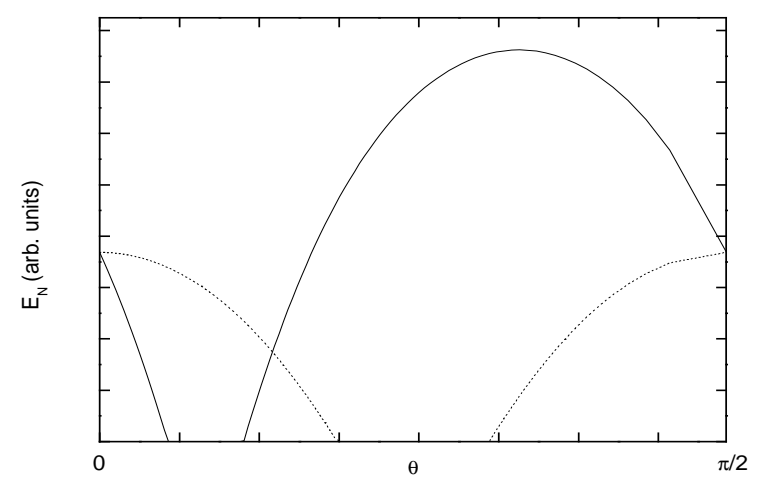

FIG. 6: Logarithmic negativity as a function of $\theta$ for a mixed symmetric state (solid line) with covariance matrix in non-standard form, compared to the one of the corresponding state with $V_{s}$ (dotted line).

On the other hand, when the mixed state are not absolutely separable there always exists, as in the pure states case, a nonvoid set of unitary modes redefinitions for which the entanglement is zero. This result, together with the previously obtained for pure states, allows one to claim that no absolutely entangled bipartite gaussian states exist. Every gaussian state can be regarded as separable, it is just a matter of choosing the right operators/modes redefinition. The maximum entanglement is reached by pure states, while mixed ones have, in general a low logarithmic negativity.
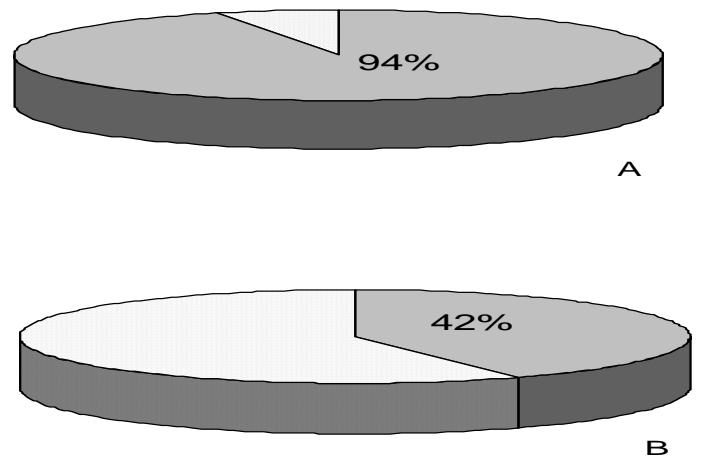

FIG. 7: Statistical results for gaussian mixed states with covariance matrix in standard form (A) and $V \neq V_{s}$ (B). The parameters range is the following: $V_{i j} \in[-1,1]$ for $i \neq j$ while $V_{i i} \in[0.5,1.5]$. The rate of absolutely separable in first case (A) states is about $94 \%$ over 7746 generated states, while in the second case (B) the rate of absolutely separable states is lower (about $42 \%$ over 4692 states).

We finally consider the case of non-symmetric mixed states for which $V \neq V_{s}$. The new statistics (Fig 7B), within the same parameters range, shows that, in comparison with the $V=V_{s}$ case, the set of states that have nonzero entanglement for some bipartition is far larger. This is due, as in the pure states case, to the fact that entanglement can be increased with a proper choice of mode redefinition and it is true even for initially separable states. We remind that a different choice of the range would lead in general to different percentage rates. 


\section{CONCLUSIONS}

The main purpose of this paper is to show how the conceptual issue of entanglement relativity i.e., the crucial dependence of entanglement on the choice of subsystems, can be addressed in a quantitative fashion for a specific, yet quite important, class of quantum states. We have studied how the entanglement of a two-modes bosonic gaussian state changes when the modes are redefined through number-conserving unitary transformations. This redefinitions have to be seen analogously to "passive" rotations in elementary geometry: the quantum state is unaffected, it is just the way it is split into subsystems i.e., modes, that changes. One obtains a set of bi-partitions parametrized by 2 by 2 unitary matrices; this makes the choice of the set of possible bipartitions, typically a huge and rather ill-defined one, easy to be dealt with analytical and numerical techniques.

We have found that with a suitable unitary redefinition of the modes every gaussian state can be regarded as separable. This means that there are no absolutely entangled bipartite gaussian states i.e., for the chosen class of tensor product structures the function $E^{-}(\rho)$ (see Eq. 1) is identically van- ishing. Moreover a large class of mixed gaussian states can be considered as absolutely separable, $E^{+}(\rho) \equiv 0$, meaning that no unitary redefinition of the modes can 'entangle' these states.

This kind of entanglement variation analysis can be extended to a larger set of Bogolioubov transformations: those that do not conserve the particle number and that are able to induce further bipartitions [22].

Of course even upon this extension the main result about the non-existence of absolutely entangled gaussian states will remain valid, whereas the set of absolutely separable states might become smaller. Finally, recent papers [16, 17] have addressed the problem of dealing with gaussian states even in fermionic systems. This naturally suggests the possibility of extending our investigations to the fermionic realm.

\section{ACKNOWLEDGMENTS.}

We are grateful to Zhao Yang, F. Illuminati, M.G.A. Paris and F. Rossi for fruitful discussions and suggestions.
[1] For reviews, see A. Steane, Rep. Prog. Phys. 61, 117 (1998); D. P. DiVincenzo and C. H. Bennett, Nature 404, 247 (2000).

[2] P. Zanardi, Phys. Rev. Lett. 87, 077901 (2001)

[3] P. Zanardi, D. A. Lidar, S. Lloyd, Phys. Rev. Lett. 92, 060402 (2004)

[4] P. Zanardi, Phys. Rev. A 65, 042101 (2002)

[5] J. Schliemann, D. Loss, and A. H. MacDonald, Phys. Rev. B 63, 085311 (2001); J. Schliemann, J. I. Cirac, M. Kuś, M. Lewenstein, and D. Loss, Phys. Rev. A 64, 022303 (2001); Y. S. Li, B. Zeng, X. S. Liu and G. L. Long, Phys. Rev. A ibid, 054302 (2001).

[6] G. Vidal, R.F. Werner, Phys. Rev. A 65032314 (2002)

[7] L.Duan, G. Giedke, J.I. Cirac, P. Zoller, Phys. Rev. Lett. 84, 2722 (2000)

[8] M.M. Wolf, J. Eisert, M.B. Plenio Phys. Rev. Lett. 90, 047904 (2003)

[9] R. Simon, Phys. Rev. Lett. 84, 2726 (2000)

[10] R. Simon, E.C.G. Sudarshan, N. Mukunda, Phys. Rev. A 37, 3028 (1988)

[11] Arvind, B. Dutta, N. Mukunda, R. Simon, quant-ph/9509002

[12] G. Adesso, A. Serafini, F. Illuminati, Phys. Rev. A 70, 022318 (2004)

[13] M. G. A. Paris, Phys. Rev. A bf59, 1615 (1999); J. Optics B 1, 299 (1999)

[14] W. Xiang-bin, Phys. Rev A 66, 024303 (2002)

[15] M.C. de Oliveira, Phys. Rev. A 70, 034303 (2004)

[16] A. Botero, B. Reznik, quant-ph/0404176

[17] P. Levay, S. Nagy, J. Pipek, quant-ph/0501145

[18] M. Kus, K. Zyczkowski, Phys. Rev A 63 032307-13 (2001)

[19] A. Peres, Phys. Rev. Lett. 77, 1413 (1996)

[20] M. Horodecki, P. Horodecki, R. Horodecki, quant-ph/9605038

[21] For this equation only, following [11], we changed the index order in the definition of $V$ by setting $x_{1}=q_{A}, x_{2}=q_{B}, x_{3}=$ $p_{A}, x_{4}=p_{B}$; in this way $S_{U}$ can be compactly defined in terms of $\mathrm{U}$.
[22] We would like to stress these number non-conserving transformations are conceptually different from the ones formerly used in this paper $(T \mathrm{~s})$. In fact the latter are merely used in the algorithm to generate new covariance matrices from each given $V_{s}$. 